\title{
A Survey of the State-of-the-Art Techniques for Cognitive Impairment Detection in the Elderly
}

\author{
Zixiang Fei ${ }^{1[0000-0003-3692-3467]}$, Erfu Yang ${ }^{1[0000-0003-1813-5950]}$, David Li ${ }^{2[0000-0002-6401-4263]}$, \\ Stephen Butler ${ }^{3[0000-0002-2103-0773]}$, Winifred Ijomah ${ }^{1}$, Neil Mackin ${ }^{4[0000-0002-5415-1395] ~}$ \\ ${ }^{1}$ Department of Design, Manufacture and Engineering Management \\ University of Strathclyde, Glasgow G1 1XJ, UK \\ \{zixiang.fei, erfu.yang, w.l.ijomah\}@strath.ac.uk \\ ${ }^{2}$ Strathclyde Institute of Pharmacy \& Biomedical Sciences \\ University of Strathclyde, Glasgow G4 ORE, UK \\ david.liestrath.ac.uk \\ ${ }^{3}$ School of Psychological Sciences and Health \\ University of Strathclyde, Glasgow G1 1QE, UK \\ stephen.butlerestrath.ac.uk \\ ${ }^{4}$ Capita plc, London SW1H 0XA, UK \\ Neil.Mackinecapita.co.uk
}

\begin{abstract}
With a growing number of elderly people in the UK, more and more of them suffer from various kinds of cognitive impairment. Cognitive impairment can be divided into different stages such as mild cognitive impairment (MCI) and severe cognitive impairment like dementia. Its early detection can be of great importance. However, it is challenging to detect cognitive impairment in the early stage with high accuracy and low cost, when most of the symptoms may not be fully expressed. This survey paper mainly reviews the state of the art techniques for the early detection of cognitive impairment and compares their advantages and weaknesses. In order to build an effective and low-cost automatic system for detecting and monitoring the cognitive impairment for a wide range of elderly people, the applications of computer vision techniques for the early detection of cognitive impairment by monitoring facial expressions, body movements and eye movements are highlighted in this paper. In additional to technique review, the main research challenges for the early detection of cognitive impairment with high accuracy and low cost are analysed in depth. Through carefully comparing and contrasting the currently popular techniques for their advantages and weaknesses, some important research directions are particularly pointed out and highlighted from the viewpoints of the authors alone.
\end{abstract}

Keywords: cognitive impairment, mild cognitive impairment (MCI), dementia, early detection, computer vision techniques, literature review 


\section{Introduction}

There are a growing number of elderly people in the UK. A significant number of people over 65 years old experience dementia. Dementia is a progressive cognitive impairment which may cause the impairment in many cognitive domains such as memory ability and language ability. Huge amounts of money is spent on the healthcare for the dementia patients every year. In addition, the number of people who have dementia is still increasing and it is estimated that there will be about 80 million dementia patients worldwide by 2040 [1]. As shown in Fig. 1, the possibility of the occurrence of dementia is often related to the age. With the age increasing, there is a higher possibility for elderly people to develop a dementia.

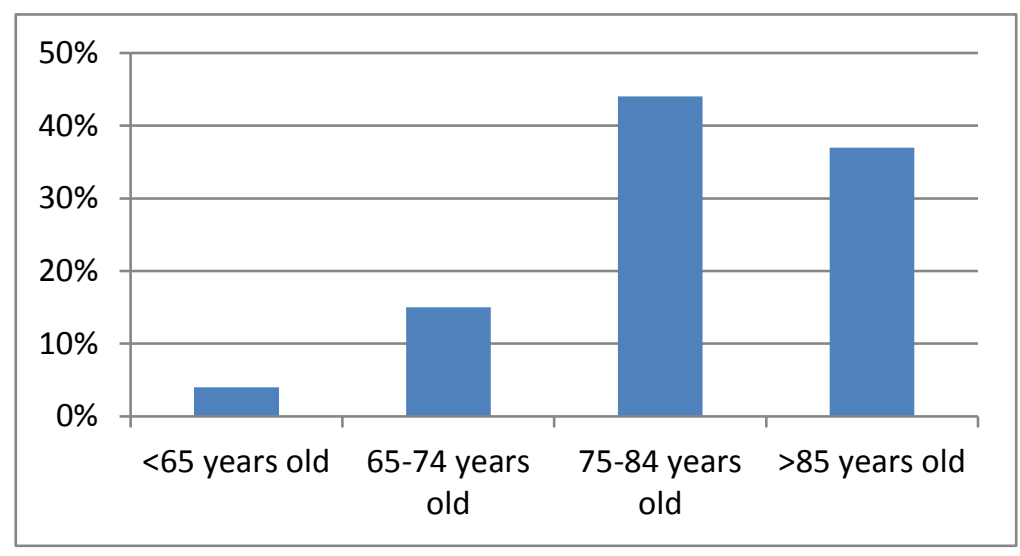

Fig. 2. the percentages of population aged 65 or over who have dementia [2]

Mild cognitive impairment (MCI) is an intermediate stage between the expected cognitive decline of normal aging and the more serious decline like dementia. For most patients with MCI, the normal activities of their daily life are not affected notably $[3,4]$. Diagnosing the MCI is similar to diagnosing mild dementia, because they have similar symptoms [5]. Currently, there are many techniques available to detect cognitive impairment including MCI and more severe cognitive impairment like dementia. Detecting the occurrence of the cognitive impairment in an early stage is of great importance.

It is critical to early detect the symptoms of cognitive impairment, monitor the progress of the disease, and provide a collective care for the elderly people with cognitive impairment, especially for those living in low-income community. However, current methods for the detection of cognitive impairment have some weaknesses and cannot achieve this goal completely. For example, some methods are not suitable for the early detection of MCI and other methods may have weakness due to the high expenses. This survey mainly describes several currently popular techniques used in the detection of cognitive impairment, compares, and contrasts their advantages and weaknesses. As shown in Fig. 2, the techniques surveyed in this paper can be generally classified into cognitive tests, neuroimaging and computer vision. The cognitive 
test techniques include instruments-based and computer-based cognitive tests. There are also two kinds of neuroimaging techniques, i.e. magnetic resonance imaging (MRI) and metabolic positron emission tomography (FDG-PET). Computer vision techniques include body motion, activities, eye movements and facial expressions.

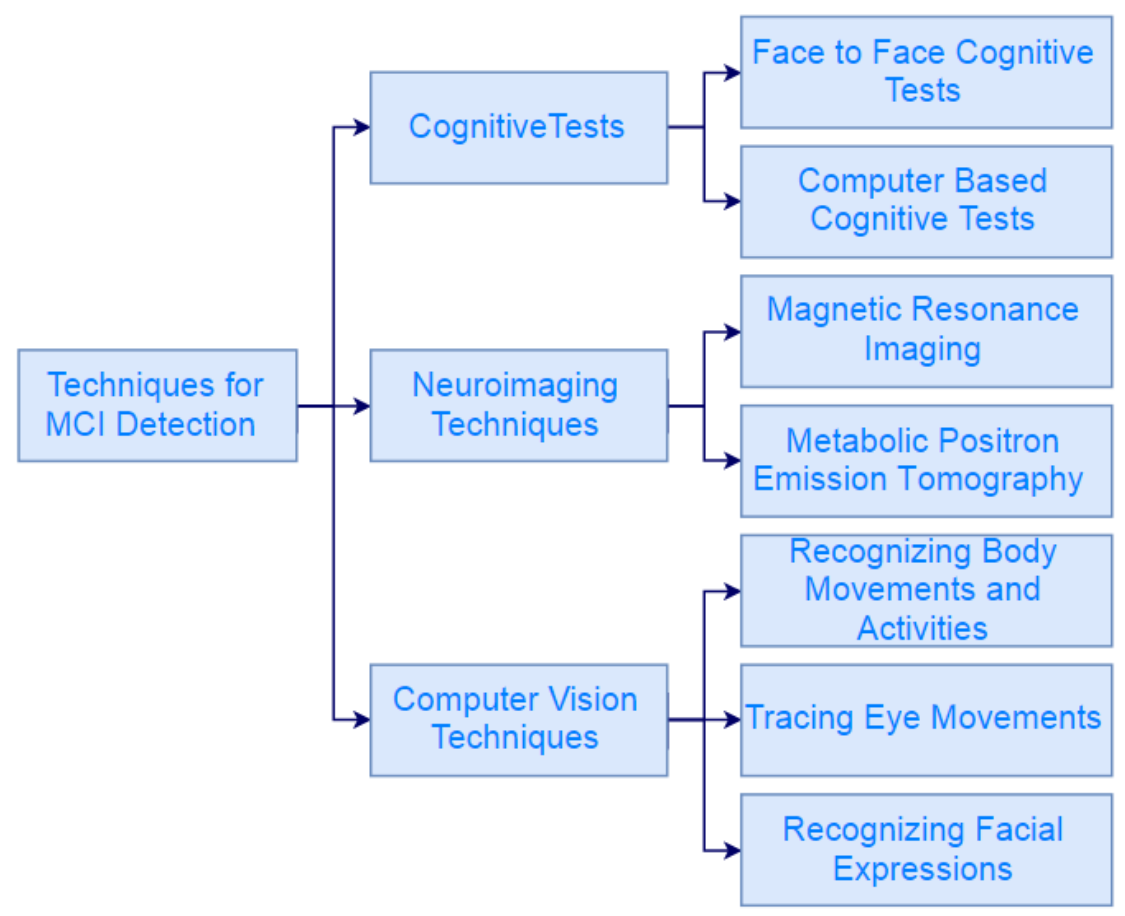

Fig. 3. Main techniques for the detection of cognitive impairment

There are also some relevant survey papers about detecting cognitive impairment. Latha et al presented a review of 22 traditional face-to-face cognitive tests which are widely used in memory clinics in the aspect of level and quality of evidence [6]. On the other hand, Katherine et al carried out a review for 18 computerized screening tools in the aspect of the test validity, reliability, comprehensiveness and usability [7]. They discussed the advantages and disadvantages of the computerized screening tools compared to the traditional face to face cognitive tests. In addition, some researchers reviewed the studies of using the magnetic resonance imaging (MRI) technique in diagnosing the cognitive impairment. The MRI technique can be used to detect the biochemical abnormalities in the regions of the brain that are related to cognitive impairment $[8,9]$. Besides, Freitas et al reviewed the studies about using the abnormalities in eye movement patterns to detect the cognitive impairment [10]. However, most of the related survey papers were focused on using one type of technique to detect cognitive impairment. In order to comprehensively review the state-of-the-art techniques currently used to detect cognitive impairment, compare and contrast their advantages and weaknesses, a literature survey is carried out in this paper. 
This paper is organized as follows. Section 1 has a general overview and sets out the outline for the literature review. The background on dementia and MCI is further introduced in Section 2. Section 3 analyses some main research challenges for the early detection of cognitive impairment with high accuracy and low cost.

The techniques of using the cognitive test to detect the cognitive impairment have been widely used in clinics. Therefore, it is detailed in Section 4. These techniques include cognitive and functional instruments, and computerized cognitive testing.

Section 5 describes the neuroimaging techniques for detecting the cognitive impairment. The techniques include the use of MRI and metabolic positron emission tomography (FDG-PET).

Computer vision techniques with body motion, activities, eye movement and facial expression are detailed in Section 6, where Section 7 is for some discussions, where some important research directions are summarised. In this part, all the methods are compared and contrasted for their advantages and weaknesses in greater details. In Section 8 , a conclusion is made to give a short summary of the paper.

\section{Background Review}

\subsection{Introduction to Dementia}

Dementia is a progressive cognitive disorder which may include the impairment in memory and other cognitive domains or skills. Dementia has some specific neuropathy logical changes, such as extracellular parenchymal lesions and intraneuronal. Because the investigation in neuropathy cannot be carried out when the patients are alive, dementia detection often involves a probabilistic diagnosis [11].

The dementia patients may have many symptoms which are different between individuals. The patients with the different types of dementia may also have different symptoms. Normally, the brain will have some changes related to the disease before the symptoms occur. For many patients, the initial symptom is that they are unable to remember new information. Then, other symptoms may appear. There are some typical symptoms for all the dementia patients such as language problem, movement problem, recognition problem, poor reasoning and judgement, and changes in personalities as well [2].

Dementia is caused by the damage of the brain cells. As a result, the brain cells cannot operate normally and communicate with each other. The affected brain parts will cause many different cognitive problems. In addition, the damage to different parts of the brain is related to the different types of dementia [12].

The common types of dementia are Alzheimer's Disease, Vascular Dementia, Dementia with Lewy Bodies and Fronto-Temporal Dementia [2]. Among them, the most common type of dementia is Alzheimer's disease. $60 \%$ to $80 \%$ of the dementia patients belong to this type of dementia [13]. 


\subsection{Mild Cognitive Impairment}

Mild cognitive impairment (MCI) is an intermediate stage between the expected cognitive decline of the normal aging and the more serious decline like dementia, which is greater than the decline expected for the individual's education and age. For the most patients with MCI, the normal activities of their daily life will not be affected greatly. Among the people with $\mathrm{MCI}$, half of them can remain stable in their cognitive situation, but other half of the people may eventually become the patients with dementia. As a result, the MCI can be considered as the risky state for dementia [3, 14]. Especially, the MCI patients with the impairment in episodic memory, verbal abilities, associative recognition impairment and visual-spatial function may have a higher risk to develop into the state of dementia $[15,16]$.

\section{$3 \quad$ Research Challenges}

Although there are many methods and techniques developed to diagnose the cognitive impairments, few of them can provide the effective and low-cost solutions for the early detection of cognitive impairments. In the following, we highlight some major research challenges on the early detection of cognitive impairment.

To begin with, because different people may have different symptoms in the early stage, it is generally difficult to diagnose the cognitive impairment in the early stage. In the MCI stage, most of the symptoms may not be fully expressed. The MCI patients may be able to live independently and act like normal people. There are only minor abnormalities or differences between the MCI patients and healthy people. In order to find the abnormalities and differences, all the cognitive domains may need to be tested such as the reaction time, the memory and attention issues and the processing speed [17].

Over the past decades, many different methods with various techniques have been developed to diagnose the severe cognitive impairment. However, they may not be sensitive enough to detect the mild cognitive decline. For example, the Mini-Mental Status Examination (MMSE) cognitive test is one of the most widely used cognitive methods to detect the severe cognitive impairment. However, it has been reported that only $18 \%$ of the MCI subjects can be detected by the MMSE cognitive test [18].

In addition, it is also a challenge to provide a low-cost and effective cognitive impairment detection solution for elderly people, especially for those living in lowincome community. For example, it is expensive to detect the cognitive impairment using the MRI-based method. Furthermore, for cognitive tests, professional neurophysiologists are needed to carry out the clinical tests for the elderly people, which suggests that a lot of time and cost are necessary. 


\section{Using Cognitive Tests to Detect the Cognitive Impairment}

\subsection{Traditional Cognitive Tests to Detect the Cognitive Impairment}

It is difficult to diagnose the MCI because of the high variability of the patients with MCI. Nowadays, the MCI is often detected by the situations reported by self or family members or by the written and task-based neurological assessments. The cognitive tests are effective and widely used in clinics. There are many kinds of cognitive screening tools such as the Mini-Mental Status Examination (MMSE), the Montreal Cognitive Assessment (MoCA), and the Functional Activities Questionnaire (FAQ). Different cognitive tests have been designed to test the cognitive impairment of various types at different stages, so the cognitive test to be used needs to be selected carefully according to the different situations.

Alex conducted a survey of 34 cases in which the cognitive tests using the MMSE were performed in clinics [19]. The MMSE cognitive test has a good accuracy for the diagnosis of cognitive impairment. From [19], it suggests that the MMSE can have a sensitivity of about $80 \%$ in detecting the cognitive impairment. However, the MMSE cognitive test has several limitations. To begin with, the MMSE cognitive test has a ceiling effect for the very mild disease such as MCI. Therefore, the MMSE cognitive test has the limited value in the diagnosis of MCI and distinguishing Alzheimer's disease against MCI. In addition, the MMSE cognitive test has a floor effect for the patients with serious cognitive impairment. Moreover, special care and adjustment are needed for the people with poor education and non-English language speaking. At last, the MMSE cognitive test also has other limitations such as its length and nonlinearity.

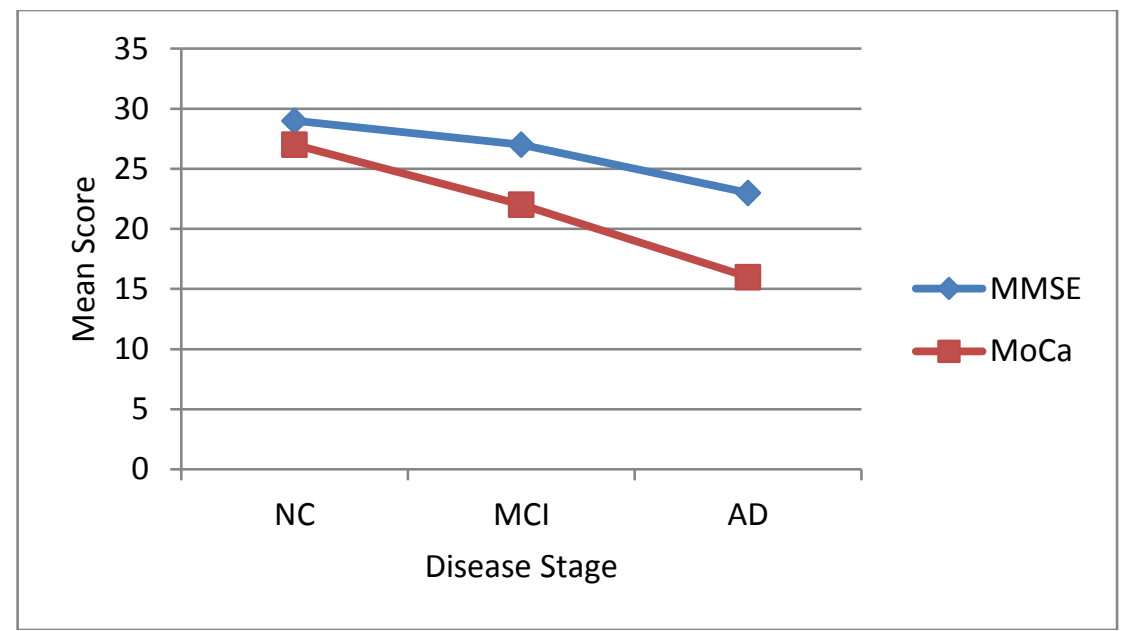

Fig. 4. Mean score for healthy control (NC), MCI patients (MCI) and dementia patients (AD) in MMSE and MoCA cognitive tests [18] 
The Montreal Cognitive Assessment (MoCA) is another cognitive screening tool which can be used to diagnose the cognitive impairment. It can often be completed in about 10 minutes. Ziad et al compared the MoCA with the MMSE in detecting the mild cognitive impairment [18]. In the experiment, there were $94 \mathrm{MCI}$ patients, 93 mild Alzheimer's disease patients and 90 healthy controls. The experiment results showed that the MoCA was better than the MMSE in detecting the MCI and mild dementia. It has been reported that the MMSE detected $18 \%$ of the MCI subjects, whereas the MoCA detected $90 \%$ of the MCI subjects. Furthermore, as shown in Fig. 3 , for the MoCA cognitive test, the difference between the mean score of the healthy controls and that of the dementia patients is bigger in comparison to the MMSE cognitive test. As a result, the MCI patients and the dementia patients may be diagnosed more easily using the MoCA cognitive test. The MoCA also had a better performance in detecting the mild dementia and excluding the elderly normal controls for about $87 \%$. The MoCA cognitive test also has more words and fewer learning trials compared to the MMSE cognitive test. In summary, the different cognitive screening tools have been designed for the different levels of the cognitive impairment. The MoCA is better to test the patients with the mild cognitive impairment and the mild dementia. However, the MMSE is designed to diagnose the more serious cognitive impairment like dementia.

Latha et al presented a review of 22 traditional face-to-face cognitive tests such as the Mini-Mental State Examination (MMSE), 6-Item Cognitive Impairment Test (6CIT) and the Hopkins Verbal Learning Test (HVLT) [6]. All these tests are designed to be completed in less than 20 minutes and are suitable for memory clinics. Latha reviewed these cognitive tests in level, quality of evidence and the types of cognitive impairment which they were suitable for. The conclusion of the review suggests that practitioners should choose the appropriate cognitive tests, because the aims of the different cognitive tests may be different from each other.

\subsection{Computer based Cognitive Tests to Detect the Cognitive Impairment}

Nowadays, many cognitive tests are also available on computers. These computer based cognitive tests are convenient and provide professional help for a wide range of people. However, they have disadvantages such as the lack of the established psychometric standards and bad user-computer interface. There are mainly two types of the computerized cognitive tests: one type is converted from the existing cognitive instruments; another type is based on the development of novel computerized screening tools [7].

Many computer based cognitive tests are converted from the existing cognitive instruments. For instance, P. Garda et al. proposed a counter propagation network based network (CPN) using the combination of neuropsychological scales which are cognitive and functional instruments such as Geriatric Depression Scale (GDS), the Minimental Status Examination (MMSE) and the Functional Activities Questionnaire (FAQ) [20]. The experiments were carried out by using the public Alzheimer's disease Neuroimage Initiative (ADNI) dataset. Kondou et al. also proposed a prototype of an automatic system for detecting the cognitive impairment [21]. The system uses the 
five-cog test, which is usually carried out manually. The system uses the image processing techniques to evaluate the cognitive functions of the users by recognizing the characters and patterns entered on a touch panel.

In order to compare and contrast the traditional cognitive tests with the computer based cognitive tests, many researchers have conducted the reviews of the existing cognitive tests and the existing computer based cognitive tests. Katherine et al presented a review for the computerized screening tools which were used to detect the cognitive situation and to diagnose the mild cognitive impairment for elderly people [7]. In the research, 18 screening tools were reviewed in the aspect of the test validity, reliability, comprehensiveness and usability.

Katherine also discussed the advantages and disadvantages of the computerized screening tools compared to the traditional cognitive tests [7]. To begin with, the computer based cognitive tests have the advantages in record accuracy, speed and sensitivity of the responses and standardized format. These kinds of the characteristics are quite important in detecting the minor changes in the cognitive situation for the patients with the mild cognitive impairment and mild dementia. In addition, compared to the traditional cognitive tests, the computerized screening tools have the advantages in cost saving and alleviating the need of professional neurophysiologists. Moreover, the computerized cognitive tests can be available from the internet and provide the support for a large population of elderly people. However, there are also some disadvantages for the computerized screening tools. For instance, one disadvantage is the lack of the established psychometric standards. In addition, some computerized screening tools may have problems in bad computer-person interface and user interactions.

Thomas made a review for the use, development and performance of some computer based cognitive tests [22]. Both of the traditional cognitive tests and computer based cognitive tests have their own characteristics. The traditional cognitive tests are more sensitive and flexible. For instance, the situations such as the moods and motivation of the patients will be taken into consideration. On the other hand, the computerized screening tools often take less time and money. The computerized screening tools are very accurate and fast, e.g., timing can be done in milliseconds. The computer based screening tools also have some disadvantages. For example, some information generated during the tests may not be interpreted correctly. Furthermore, the results obtained from the computer based screening tools are not very sensitive. In other words, if someone does well in the computer based tests, it means that he does not have cognitive problems. However, someone does not do well in the computer based tests cannot make sure that he has dementia or other cognitive diseases. Therefore, clinical diagnosis may still be needed to accurately diagnose.

\section{$5 \quad$ Using Neuroimaging Techniques to Diagnose Cognitive Impairment}

There are two kinds of neuroimaging techniques which are widely used in clinics for the early detection of the cognitive impairment, i.e. magnetic resonance imaging 
(MRI) and metabolic positron emission tomography (FDG-PET) [23]. These two techniques are both used to detect the abnormalities in brain regions for the early detection of the patient with the cognitive impairment. Because the brain of the patient with the cognitive impairment may have the abnormalities before the symptoms appear, neuroimaging is quite useful for the early detection of the cognitive impairment when the clinical symptoms have not fully expressed [2]. However, diagnosing the cognitive impairment is expensive and unfriendly to those living in low-income community.

Herholz et al presented a novel indicator of the FDG-PET with the automated voxel-based procedure to diagnose the cognitive impairment [24]. The data was collected from large amounts of the participants consisting of 110 normal healthy controls and 395 patients with the cognitive impairment. The results showed that all the regions of the brain related to the cognitive impairment were affected in the beginning of the disease.

On the other hand, many researchers reviewed the studies of using the MRI in diagnosing the cognitive impairment. Yuanyu et al conducted a review of the studies about the magnetic resonance imaging [9]. The MRI technique can be used to detect the biochemical abnormalities in the regions of the brain that are related to the cognitive impairment. As a result, the MRI can be used for the early detection of the cognitive impairment and monitoring the disease progress. Amy et al also reviewed the studies about the relationship between the cognitive problems with the neurometabolite values [8]. They have made a conclusion that the magnetic resonance spectroscopy (MRS) can be used to note the longitudinal changes and detect the cognitive problems such as dementia in an early stage.

To develop the MRI techniques in the diagnosis of the cognitive impairment, Stefan et al proposed a novel automatic system to diagnose the cognitive impairment [25]. The system could be used to distinguish the normal aging from the cognitive impairment. In this research, the support vector machine (SVM) was used in MRI to detect the different disease states. The experiment showed that $96 \%$ of the patients were classified for the disease types correctly using the brain images.

\section{Using Computer Vision to Detect the Cognitive Impairment}

\subsection{Detecting the Cognitive Impairment with Eye Movement}

Abnormalities in eye movement patterns may be associated with the deficits in the patients with the cognitive impairment. As a result, the patients with the cognitive impairment can be diagnosed by analysing their eye movements. The patients with the cognitive impairment may have the deficits in static spatial contrast sensitivity, visual attention, shape-from-motion, colour, visual spatial construction, and visual memory. In addition, some researchers have found that several characteristics of the eye movement patterns may be particularly useful to diagnose the cognitive impairment. These characteristics are such as fixation duration, re-fixations, saccade orientation, pupil diameter smooth, pursuit movements and saccadic inhibition [10, 26]. They have also designed experiments to detect the cognitive impairment by eye movements 
[27-29]. However, most of the related investigations are carried out in dementia patients and the investigations in MCI patients are insufficient [10]. Furthermore, to improve the precision in measurements, eye trackers are often needed.

Yanxia et al worked on the linkage between the cognitive situations obtained from the cognitive tests and the eye movement patterns collected when the participants were watching videos. They tried to find the specific eye movement patterns which were related to the cognitive impairment and then used these eye movement patterns for the automatic cognitive assessment [27]. From the experiments, they found that the participants with the cognitive impairment would have slower saccade motion and longer fixation time on average. However, the results might be limited because of the small number of samples obtained during the experiments.

In order to obtain the eye movements, eye trackers are often used to record the eye movement patterns while the patients with the cognitive impairment are doing some tasks such as watching images or videos on computers [10].

In particular, there are some eye movement experiments used to detect the cognitive impairment. One possible experiment example is the visual paired comparison (VPC) task. In the experiment, the participant is asked to select the picture which she saw previously. The eye tracker will be used to track the eye movement when the participant is doing this task. The eye movement pattern will be used to diagnose if the participant has a cognitive impairment or not.

Some researchers used the VPC task to find the difference in the eye movements between the patients with the cognitive impairment and the normal people. For instance, Dmitry et al used the VPC task to detect the memory impairment in the MCI patients [28]. The VPC task would test the participants if they could figure out the novel visual stimuli with the repeated visual stimuli. As a result, the healthy controls would watch the novel images for more time.

Gerardo et al proposed a novel method to detect the cognitive problems by the eye movements in the activities of reading proverbs [29]. In the experiments, there were 20 participants with Alzheimer's disease and 40 healthy participants. By the experiments, they have found that compared with the participants with Alzheimer's disease, the healthy participants would have the longer gaze periods for words with predictability. It showed that the participants with Alzheimer's disease had the impairment in word predictions.

\subsection{Detecting the Cognitive Impairment by Recognizing Activities}

When the cognitive decline occurs, it will have the impacts on doing their daily activities for elderly people [30]. As a result, the recognition of their activities has the potential for the detection of cognitive impairment. In particular, the systems with these techniques can be used to monitor the cognitive changes and disease progress for the elderly people automatically.

Sabrina et al. proposed a RGBD camera monitoring system to monitor the patients with the cognitive impairment using neural networks. The system can monitor the patients in home environment during daily activities which are in the Direct Assessment of Functional Status (DAFS) index. DAFS proposed by Zanetti et al is a stand- 
ardized observation-based checklist that is used to evaluate the cognitive situation for the elderly people with cognitive impairment [31].The system was focused on monitoring the activities of brushing teeth and grooming hair. The system could detect the abnormalities during the activities to find out the cognitive situation of the elderly people. The result of the experiment showed that the system was able to recognize the daily activities and calculate the DAFS score to detect the occurrence of the cognitive decline. There were some problems that it could not recognize the objects during the activities and the kinds of the activities could be recognized was limited.

Aleksandar et al also proposed a system to monitor the cognitive decline by recognizing the activities using the fusion of machine vision technique and RPID technique [32]. The system could provide an early warning of the disease by analysing the changes in their behavioural patterns during daily activities. The result for monitoring the patients would be provided to caregivers or doctors. This system could reduce the workload of the caregivers by monitoring the cognitive situations of the patients automatically. In [32], cooking and memory card game were selected to be as two daily activities monitored. However, this research was still in progress and there was no experiments carried out yet.

The kinds of activities could be recognized were limited in the both of the cases above. The various kinds of daily activities should be able to be monitored and recognized to know the cognitive situation of the elderly people in order that the MCI could be detected at the beginning. Vincent et al. proposed a system which could recognize 8 kinds of activities for the people with the cognitive impairment [33]. The activities were such as feeding birds, preparing and eating meal, making tea, playing a $\mathrm{CD}$ and so on. This system recognized the activities using the videos recorded by wearable devices. It was made up of the fusion of the object detectors and the location detectors. For example, to detect the activities of preparing and eating meal, the objects such as bowls, spoons and food were involved and the place such as kitchen was involved. The overall performance showed a good result. However, the recognition accuracy for some activities was low such as the recognition of making a phone call. The reason was that the phone was always near the ear and the wearable device might not be able to detect the phone. In addition, making phone calls might happen in many different places.

In order to improve the accuracy for recognizing the activities in complex environment and in long videos, locating the place and time that the activities take place is important. Konstantinos et al did a research on monitoring and recognizing the activities for the elderly people [34]. The research focused on the improvement of the performance for recognizing the Activities of Daily Living (ADL). They proposed a novel algorithm that would locate the Activities of Daily Living both in time and space. It would find the start and end time of the activities and where they took place.

It may be difficult to monitor the patients during their daily activities all the day to find the abnormal body movements. In spite of monitoring the patients in daily activities, a method for detecting the cognitive problem when playing an interactive physical game was proposed in [35]. The cognitive situation can be evaluated by the video recorded with the body motion sensor when the elderly people are playing the game. The system can record the parameters of the body motion. In [35], 5 kinds of games 
were developed using the Kinect to detect if the elderly people had problems of the mild cognitive impairment.

\subsection{Detecting the Cognitive Impairment by Facial Expression}

The people with the cognitive impairment have the impairment in recognizing the facial expressions [36-38]. Meanwhile, the facial expressions of the people with the cognitive impairment also show the difference compared to normal people. As a result, the facial expressions also have potential to diagnose the cognitive impairment by observing and monitoring the facial expressions. However, there are few investigations in detecting the cognitive impairment by monitoring the facial expressions.

The difference of the facial expressions between the patients with the cognitive impairment and the normal people was discussed in many research papers [39, 40]. Keith et al researched on the facial muscle activities for the patients with Alzheimer's Diseases (AD) [39]. In the experiment, the groups of patients with the AD and the groups of the normal controls were asked to watch some emotion-eliciting images while the facial muscles were researched. Smiles are often related to the zygomatic activities and frowns are often related to the corrugator activities. The experiment results showed that the $\mathrm{AD}$ patients showed more corrugator activities when viewing the negative images. Furthermore, there was also a big difference for zygomatic activities between the AD patients and the normal people. As shown in Fig. 4, the AD patients showed the maximum zygomatic activities when they were watching the negative images and minimum activities for the positive ones.

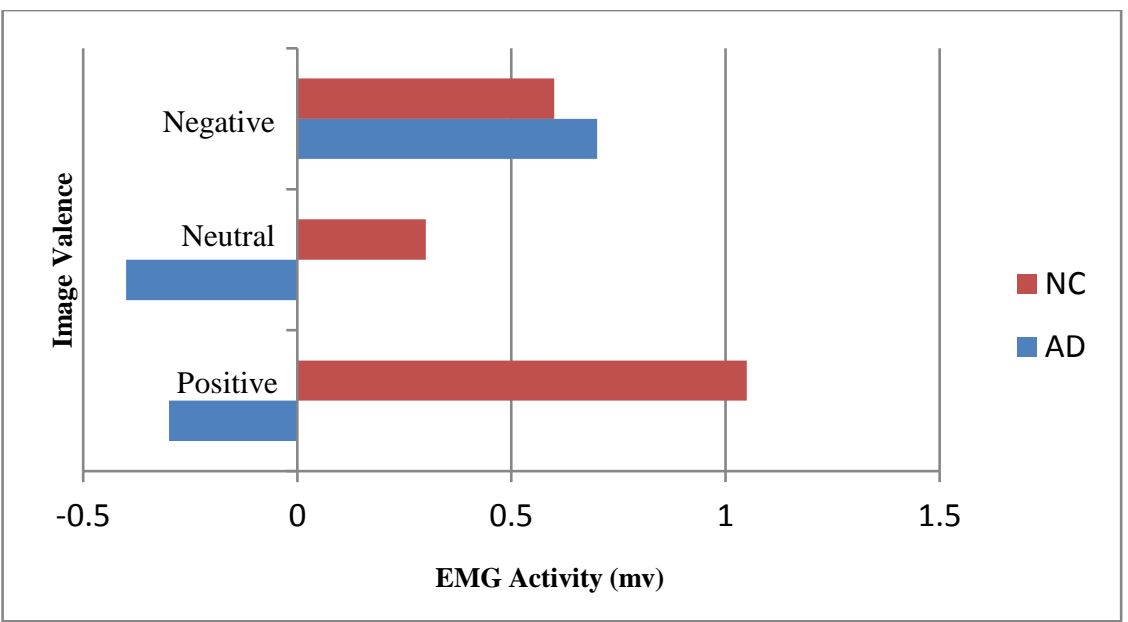

Fig. 5. Zygomatic change scores when viewing positive, neutral and negative images for the normal control (NC) and the patients with Alzheimer's Diseases (AD) [39]

The patients with the cognitive impairment have the impairment to control facial muscles and to express their feelings. Julie et al researched on the facial expressions of the patients with the cognitive impairment [40]. In the experiment, the patients with 
the cognitive impairment and normal controls were asked to watch videos, when the facial expressions were recorded. In addition, they were asked to express their feelings in the conditions of spontaneous expressions, amplification and suppression of emotions. The results showed that for the patients with the cognitive impairment, the behavioural amplification of the expressed emotion was affected. However, the subjective feelings and suppression of emotions were not affected.

However, there are also some difficulties to use the facial expressions to diagnose the cognitive impairment. To begin with, the facial expressions may be different between individuals and would be affected by the premorbid personality [41]. The influence of the premorbid personality on the facial expressions was discussed in Carol's paper. They used the research data of the facial expressions collected during family visits to the patients with the cognitive impairment. They found that the avoidant attached individuals would show less the positive facial expressions than the securely attached individuals. In addition, the patients with the premorbid hostility would show more negative facial expressions. Moreover, the facial expression behaviours would be affected by culture and were different between the western people and the eastern people [42].

Furthermore, the facial expressions of the patients with the cognitive impairment will be affected by apathy [43]. Ulrich pointed out that with the progression of the disease, the total facial expressions would not decrease. Instead, the total facial expressions would increase, but there would be less specific facial expressions.

In order to use the facial expressions to diagnose the cognitive impairment automatically, computer vision technologies will play an important role in this field. There are many good survey papers about dealing with the facial expressions problems using computer vision techniques [44-46]. They mainly compared and contrasted the different techniques to carry out the major steps for the facial expression recognition such as face localization, face registration, face representation, face features extraction and face expression recognition.

For instance, Basel et al reviewed the studies of the facial expression recognition and paid attention to the sections such as face normalization, dynamics and intensity for the facial expressions [44]. Georgia et al mainly reviewed the computer vision techniques for the facial expression in the aspects of 3D or 4D facial expressions [45]. Evangelos et al reviewed the state of the art computer vision techniques for the facial expressions and laid the emphasis on the part of facial representation [46]. In addition, they focused on discussing the difficulties in the process of the facial expression recognition such as the problems related to illuminations, the variations in head position, the errors in registration and so on.

Meanwhile, many researchers worked on the improvement of the automatic facial expression recognition techniques. For instance, Andre et al. proposed a novel solution for the recognition of expressions using a convolutional neural network and applying the pre-processing techniques to extract the specific features [47]. Murari et al. proposed a method to recognize the facial expressions by extracting the facial features using the higher-order Zernike moments and classification with an ANN based classifier [48]. Ashok et al. had a survey on the automatic recognition of the facial expressions and compared with the human visual system that deals with the same problem 
[49]. Some technologies such as deep learning will also improve the accuracy of the facial expression recognition [50].

\section{$7 \quad$ Discussion and Research Directions}

In this paper, many current methods and techniques used to diagnose and detect the cognitive impairment have been reviewed. There have been some big changes and progresses in the field of detecting and diagnosing the cognitive impairment in recent years. However, all these existing techniques have their own weaknesses. The current methods for diagnosing the cognitive impairment are still unsatisfying [51]. New techniques in particular need to be developed to meet the needs of the early detection of the cognitive impairment with the high accuracy and low cost.

Regarding the weaknesses of the existing techniques, both the traditional face to face cognitive tests and computer-based cognitive tests have the problems with diagnosing the mild cognitive impairment. More specifically, some personal information like age, education and personality will influence the test result and need to be taken into consideration carefully [52]. Moreover, the different cognitive tests are often suitable for the different types and stages of the cognitive impairment. As a result, the cognitive test to be used needs to be selected carefully according to the different situations. In addition, for the traditional face to face cognitive tests, the professional neurophysiologists are needed to carry out the cognitive tests for the patients. Meanwhile, for the computer based cognitive tests, if the elderly people would like to take the tests on their own, some basic computer skills are often required.

Although diagnosing the cognitive impairment with the neuroimaging techniques are widely used in clinics, the major weakness of them is the high expenses. Besides, the neuroimaging techniques are often used to diagnose the cognitive impairment only when the patients are found that they have serious cognitive problems.

Diagnosing the cognitive impairment by eye movements, facial expressions and daily activities are still in developing stage. Using the eye movements to diagnose the cognitive impairment needs eye trackers to improve the precision in measurements. In addition, the effective eye movement experiments need to be designed precisely. Meanwhile, the existing systems to detect the cognitive situations by the recognition of the activities are not effective and intelligent enough and the types of activities can be recognized are very limited. Besides, the systems which can detect the cognitive impairment by using facial expressions are still in progress. The major characteristics of these techniques are also compared and contrasted in Table 1.

Table 1. Compare and contrast several techniques to detect the cognitive impairment

\begin{tabular}{|c|c|c|c|c|}
\hline Techniques & Characteristics & Weaknesses & Setting & Cost \\
\hline $\begin{array}{c}\text { Face to Face } \\
\text { Cognitive } \\
\text { Tests }\end{array}$ & $\begin{array}{l}\text { Test the cognitive do- } \\
\text { mains such as memory } \\
\text { ability } \\
\text { Finish in less than } 20 \\
\text { minutes }\end{array}$ & $\begin{array}{l}\text { Unfriendly to people with } \\
\text { poor education back- } \\
\text { ground } \\
\text { Dependent on profession- } \\
\text { al neurophysiologists }\end{array}$ & $\begin{array}{l}\text { Memory } \\
\text { clinics }\end{array}$ & Medium \\
\hline
\end{tabular}




\begin{tabular}{|c|c|c|c|c|}
\hline $\begin{array}{c}\text { Computer } \\
\text { Based Cogni- } \\
\text { tive Tests }\end{array}$ & $\begin{array}{c}\text { Test several cognitive } \\
\text { domains Available } \\
\text { through internet }\end{array}$ & $\begin{array}{c}\text { Requirement of computer } \\
\text { skills } \\
\text { Problems from poor } \\
\text { computer-human inter- } \\
\text { face }\end{array}$ & Homes & $\begin{array}{c}\text { Low or } \\
\text { free }\end{array}$ \\
\hline $\begin{array}{c}\text { Neuroimaging } \\
\text { Techniques }\end{array}$ & $\begin{array}{c}\text { Able to detect the ab- } \\
\text { normality in the brain } \\
\text { when clinical symptoms } \\
\text { are not fully expressed }\end{array}$ & $\begin{array}{c}\text { Suitable for detailed } \\
\text { diagnosis, not for early } \\
\text { detection }\end{array}$ & Clinics & High \\
\hline $\begin{array}{c}\text { Recognising } \\
\text { Body Move- } \\
\text { ments and } \\
\text { Activities }\end{array}$ & $\begin{array}{c}\text { Able to detect the cogni- } \\
\text { tive decline }\end{array}$ & $\begin{array}{c}\text { Limited types of activities } \\
\text { that can be recognised } \\
\text { Difficulties in detecting } \\
\text { minor abnormalities }\end{array}$ & Homes & $\begin{array}{c}\text { Low or } \\
\text { free }\end{array}$ \\
\hline $\begin{array}{c}\text { Tracing Eye } \\
\text { Movements }\end{array}$ & $\begin{array}{c}\text { Able to detect deficits in tracker } \\
\text { visual attention and so on } \\
\text { Need improvements in } \\
\text { effective eye movement } \\
\text { experiments }\end{array}$ & Labs & \begin{tabular}{c} 
Medium \\
\hline $\begin{array}{c}\text { Recognizing } \\
\text { Facial Ex- } \\
\text { pressions }\end{array}$
\end{tabular} Notice facial abnormali- \\
ties & No developed system & Homes & $\begin{array}{c}\text { Low or } \\
\text { free }\end{array}$ \\
\hline
\end{tabular}

Regarding the research directions, they can be roughly divided into three aspects: effective cognitive tests, advanced neuroimaging techniques for clinics situations, and automatic cognitive impairment detection system for family use.

The automatic cognitive impairment detection systems are suitable for the use in home situations for the early detection of the cognitive impairment and monitoring the disease progress. The systems need to be low cost, convenient and user friendly so that the systems can be used widely, but it may not be very precise as devices in clinics. The system should be able to detect the abnormalities related to the cognitive problems. The cameras from mobile phones could be used to monitor the eye movements, facial expressions and activities of elderly people. The system can work as an app on the mobile phones.

The future direction of the cognitive tests will probably be computer based cognitive tests, which can be easily available from internet. The input methods of the computer based cognitive tests will not be limited to keyboards and touch panels but will also include cameras which can record the eye movement, facial expression and body movement. The cognitive tests need to be able to diagnose the cognitive situations for the different types and stages of the cognitive impairment and can be completed in several minutes.

As the most direct and effective methods to diagnose the cognitive impairment in clinics, the future direction of the neuroimaging techniques should have a high accuracy and low cost. In addition, the effective automatic system to diagnose the cognitive impairment with the neuroimaging techniques needs to be developed. 


\section{Conclusion}

This survey paper has reviewed several currently popular techniques for the early detection of cognitive impairment and discussed their advantages and weaknesses. These techniques included cognitive test, neuroimaging and computer vision. The cognitive test techniques had the traditional face to face cognitive tests and computerized cognitive tests. Two kinds of neuroimaging techniques were reviewed, i.e., magnetic resonance imaging (MRI) and metabolic positron emission tomography (FDGPET). Computer vision techniques with body motion, eye movement and facial expression were highlighted as they have potentials to build cost-effective solutions for early detection of cognitive impairments.

This paper has also analysed and discussed about the future research directions in the area of diagnosing and early detecting the cognitive impairment. The first future direction is about the automatic cognitive impairment detection systems that use multiple information sources such as eye movements, facial expressions and body motions. These systems are expected to monitor the progress of the disease automatically and they are suitable for family use. The second future direction is about the advanced neuroimaging techniques to diagnose the different types of cognitive impairment with a higher accuracy and low cost for clinic situations. The third direction is about the improvement of the existing cognitive tests which can be carried out regularly with low cost and be easily available for the elderly living in the low-income communities.

\section{Acknowledgements}

This research is funded by Strathclyde's Strategic Technology Partnership (STP) Programme with CAPITA (2016-2019). The authors thank Dr Neil Mackin (CAPITA mentor) and Miss Angela Anderson (the STP's coordinator) for their support. The contents including any opinions and conclusions made in this paper are those of the authors alone. They do not necessarily represent the views of CAPITA plc.

\section{References}

1. Ferri, C.P., Prince, M., Brayne, C., Brodaty, H., Fratiglioni, L., Ganguli, M., Hall, K., Hasegawa, K., Hendrie, H., Huang, Y., Jorm, A., Mathers, C., Menezes, P.R., Rimmer, E., Scazufca, M.: Global prevalence of dementia: A Delphi consensus study. Lancet. 366, (2005). doi:10.1016/S0140-6736(05)67889-0

2. Gaugler, J., James, B., Johnson, T., Scholz, K., Weuve, J.: 2016 Alzheimer's disease facts and figures. Alzheimer's Dement. 12, (2016). doi:10.1016/j.jalz.2016.03.001

3. Wu, N.S.C., Ho, K.-S.: Mild cognitive impairment. Hong Kong Pract. 31, (2009)

4. Winblad, B., Palmer, K., Kivipelto, M., Jelic, V., Fratiglioni, L., Wahlund, L.-O., Nordberg, A., Bäckman, L., Albert, M., Almkvist, O., Arai, H., Basun, H., Blennow, K., De Leon, M., Decarli, C., Erkinjuntti, T., Giacobini, E., Graff, C., Hardy, J., Jack, C., Jorm, A., Ritchie, K., Van Duijn, C., Visser, P., Petersen, R.C.: Mild cognitive 
impairment - Beyond controversies, towards a consensus: Report of the International Working Group on Mild Cognitive Impairment. J. Intern. Med. 256, (2004). doi:10.1111/j.1365-2796.2004.01380.x

5. Petersen, R.C.: Mild cognitive impairment as a diagnostic entity. J. Intern. Med. 256, (2004). doi:10.1111/j.1365-2796.2004.01388.x

6. Velayudhan, L., Ryu, S.-H., Raczek, M., Philpot, M., Lindesay, J., Critchfield, M., Livingston, G.: Review of brief cognitive tests for patients with suspected dementia. Int. Psychogeriatrics. 26, (2014). doi:10.1017/S1041610214000416

7. Wild, K., Howieson, D., Webbe, F., Seelye, A., Kaye, J.: Status of computerized cognitive testing in aging: A systematic review. Alzheimer's Dement. 4, (2008). doi:10.1016/j.jalz.2008.07.003

8. Ross, A.J., Sachdev, P.S.: Magnetic resonance spectroscopy in cognitive research. Brain Res. Rev. 44, (2004). doi:10.1016/j.brainresrev.2003.11.001

9. Hsu, Y.-Y., Du, A.-T., Schuff, N., Weiner, M.W.: Magnetic resonance imaging and magnetic resonance spectroscopy in dementias. J. Geriatr. Psychiatry Neurol. 14, (2001)

10. Pereira, M.L.F., Von Zuben A Camargo, M., Aprahamian, I., V Forlenza, O.: Eye movement analysis and cognitive processing: detecting indicators of conversion to Alzheimer ' s disease. Neuropsychiatr. Dis. Treat. 10, 1273-1285 (2014). doi:10.2147/NDT.S55371

11. Dubois, B., Feldman, H.H., Jacova, C., Cummings, J.L., DeKosky, S.T., BarbergerGateau, P., Delacourte, A., Frisoni, G., Fox, N.C., Galasko, D., Gauthier, S., Hampel, H., Jicha, G.A., Meguro, K., O’Brien, J., Pasquier, F., Robert, P., Rossor, M., Salloway, S., Sarazin, M., de Souza, L.C., Stern, Y., Visser, P.J., Scheltens, P.: Revising the definition of Alzheimer's disease: A new lexicon. Lancet Neurol. 9, (2010). doi:10.1016/S1474-4422(10)70223-4

12. Dementia - Signs, Symptoms, Causes, Tests, Treatment, Care | alz.org, http://www.alz.org/what-is-dementia.asp

13. Blennow, K., de Leon, M.J., Zetterberg, H.: Alzheimer's disease. Lancet. 368, (2006). doi:10.1016/S0140-6736(06)69113-7

14. Nestor, P.J., Scheltens, P., Hodges, J.R.: Advances in the early detection of alzheimer's disease. Nat. Rev. Neurosci. 10, (2004). doi:10.1038/nrn1433

15. Arnáiz, E., Almkvist, O.: Neuropsychological features of mild cognitive impairment and preclinical Alzheimer's disease. Acta Neurol. Scand. Suppl. 107, (2003)

16. Troyer, A.K., Murphy, K.J., Anderson, N.D., Craik, F.I.M., Moscovitch, M., Maione, A., Gao, F.: Associative recognition in mild cognitive impairment: Relationship to hippocampal volume and apolipoprotein E. Neuropsychologia. 50, (2012). doi:10.1016/j.neuropsychologia.2012.10.018

17. Gualtieri, C.T.: Dementia screening in light of the diversity of the condition. J. Insur. Med. 36, (2004)

18. Nasreddine, Z.S., Phillips, N.A., Bédirian, V., Charbonneau, S., Whitehead, V., Collin, I., Cummings, J.L., Chertkow, H.: The Montreal Cognitive Assessment, MoCA: A brief screening tool for mild cognitive impairment. J. Am. Geriatr. Soc. 53, (2005). doi:10.1111/j.1532-5415.2005.53221.x

19. Mitchell, A.J.: A meta-analysis of the accuracy of the mini-mental state examination in 
the detection of dementia and mild cognitive impairment. J. Psychiatr. Res. 43, (2009). doi:10.1016/j.jpsychires.2008.04.014

20. Báez, P.G., Viadero, C.F., Espinosa, N.R., Pérez Del Pino, M.A., Suárez-Araujo, C.P.: Detection of mild cognitive impairment using a counterpropagation network based system. An e-health solution. In: 2015 International Workshop on Computational Intelligence for Multimedia Understanding, IWCIM 2015 (2015)

21. Kondou, Y., Kawasumi, M., Yamamoto, O., Yamada, M., Yamamoto, S., Nakanno, T.: Study of early screening method of dementia and its systemization. In: Proceedings of the 11th IAPR Conference on Machine Vision Applications, MVA 2009 (2009)

22. Gualtieri, C.T.: Dementia screening using computerized tests. J. Insur. Med. 36, (2004)

23. Mosconi, L., Brys, M., Glodzik-Sobanska, L., De Santi, S., Rusinek, H., de Leon, M.J.: Early detection of Alzheimer's disease using neuroimaging. Exp. Gerontol. 42, (2007). doi:10.1016/j.exger.2006.05.016

24. Herholz, K., Salmon, E., Perani, D., Baron, J.-C., Holthoff, V., Frölich, L., Schönknecht, P., Ito, K., Mielke, R., Kalbe, E., Zündorf, G., Delbeuck, X., Pelati, O., Anchisi, D., Fazio, F., Kerrouche, N., Desgranges, B., Eustache, F., BeuthienBaumann, B., Menzel, C., Schröder, J., Kato, T., Arahata, Y., Henze, M., Heiss, W.D.: Discrimination between Alzheimer dementia and controls by automated analysis of multicenter FDG PET. Neuroimage. 17, (2002). doi:10.1006/nimg.2002.1208

25. Klöppel, S., Stonnington, C.M., Chu, C., Draganski, B., Scahill, R.I., Rohrer, J.D., Fox, N.C., Jack Jr., C.R., Ashburner, J., Frackowiak, R.S.J.: Automatic classification of MR scans in Alzheimer's disease. Brain. 131, (2008). doi:10.1093/brain/awm319

26. MacAskill, M.R., Anderson, T.J.: Eye movements in neurodegenerative diseases. Curr. Opin. Neurol. 29, 61-68 (2016). doi:10.1097/WCO.0000000000000274

27. Zhang, Y., Wilcockson, T., Kim, K.I., Crawford, T., Gellersen, H., Sawyer, P.: Monitoring dementia with automatic eye movements analysis. (2016)

28. Lagun, D., Manzanares, C., Zola, S.M., Buffalo, E.A., Agichtein, E.: Detecting cognitive impairment by eye movement analysis using automatic classification algorithms. J. Neurosci. Methods. 201, (2011). doi:10.1016/j.jneumeth.2011.06.027

29. Fernández, G., Castro, L.R., Schumacher, M., Agamennoni, O.E.: Diagnosis of mild Alzheimer disease through the analysis of eye movements during reading. J. Integr. Neurosci. 14, (2015). doi:10.1142/S0219635215500090

30. Iarlori, S., Ferracuti, F., Giantomassi, A., Longhi, S.: RGBD camera monitoring system for Alzheimer's disease assessment using Recurrent Neural Networks with Parametric Bias action recognition. In: IFAC Proceedings Volumes (IFACPapersOnline) (2014)

31. Zanetti, O., Frisoni, G.B., Rozzini, L., Bianchetti, A., Trabucchi, M.: Validity of direct assessment of functional status as a tool for measuring Alzheimer's disease severity. Age Ageing. 27, (1998). doi:10.1093/ageing/27.5.615

32. Matic, A., Osmani, V.: Technologies to monitor cognitive decline: A preliminary case study. In: 2009 3rd International Conference on Pervasive Computing Technologies for Healthcare - Pervasive Health 2009, PCTHealth 2009 (2009)

33. Buso, V., Hopper, L., Benois-Pineau, J., Plans, P.-M., Megret, R.: Recognition of Activities of Daily Living in natural "at home" scenario for assessment of Alzheimer's 
disease patients. In: 2015 IEEE International Conference on Multimedia and Expo Workshops, ICMEW 2015 (2015)

34. Avgerinakis, K., Briassouli, A., Kompatsiaris, I.: Activity detection and recognition of daily living events. In: MIIRH 2013 - Proceedings of the 1st ACM International Workshop on Multimedia Indexing and Information Retrieval for Heathcare, Colocated with ACM Multimedia 2013 (2013)

35. Chen, Y.T., Hou, C.J., Huang, M.W., Dong, J.H., Zhou, J.Y., Hung, I.C.: The design of interactive physical game for cognitive ability detecting for elderly with mild cognitive impairment. In: IFMBE Proceedings (2015)

36. Sapey-Triomphe, L.-A., Heckemann, R.A., Boublay, N., Dorey, J.-M., Hénaff, M.-A., Rouch, I., Padovan, C., Hammers, A., Krolak-Salmon, P.: Neuroanatomical correlates of recognizing face expressions in mild stages of Alzheimer's disease. PLoS One. 10, (2015). doi:10.1371/journal.pone.0143586

37. Yang, L., Zhao, X., Wang, L., Yu, L., Song, M., Wang, X.: Emotional face recognition deficit in amnestic patients with mild cognitive impairment: Behavioral and electrophysiological evidence. Neuropsychiatr. Dis. Treat. 11, (2015). doi:10.2147/NDT.S85169

38. Varjassyová, A., Hoř́nek, D., Andel, R., Amlerova, J., Laczó, J., Sheardová, K., Magerová, H., Holmerová, I., Vyhnálek, M., Bradáč, O., Geda, Y.E., Hort, J.: Recognition of facial emotional expression in amnestic mild cognitive impairment. J. Alzheimer's Dis. 33, (2013). doi:10.3233/JAD-2012-120148

39. Burton, K., Kaszniak, A.: Emotional experience and facial expression in Alzheimer's disease. Aging, Neuropsychol. Cogn. 13, (2006). doi:10.1080/13825580600735085

40. Henry, J.D., Rendell, P.G., Scicluna, A., Jackson, M., Phillips, L.H.: Emotion Experience, Expression, and Regulation in Alzheimer's Disease. Psychol. Aging. 24, (2009). doi:10.1037/a0014001

41. Magai, C., Cohen, C.I., Culver, C., Gomberg, D., Malatesta, C.: Relation between premorbid personality and patterns of emotion expression in mid- to late-stage dementia. Int. J. Geriatr. Psychiatry. 12, (1997). doi:10.1002/(SICI)10991166(199711)12:11\&lt;1092::AID-GPS690\&gt;3.0.CO;2-X

42. Jack, R.E., Garrod, O.G.B., Yu, H., Caldara, R., Schyns, P.G.: Facial expressions of emotion are not culturally universal. Proc. Natl. Acad. Sci. U. S. A. 109, (2012). doi:10.1073/pnas.1200155109

43. Seidl, U., Lueken, U., Thomann, P.A., Kruse, A., Schröder, J.: Facial expression in Alzheimer's disease: Impact of cognitive deficits and neuropsychiatric symptoms. Am. J. Alzheimers. Dis. Other Demen. 27, (2012). doi:10.1177/1533317512440495

44. Fasel, B., Luettin, J.: Automatic facial expression analysis: A survey. Pattern Recognit. 36, (2003). doi:10.1016/S0031-3203(02)00052-3

45. Sandbach, G., Zafeiriou, S., Pantic, M., Yin, L.: Static and dynamic 3D facial expression recognition: A comprehensive survey. Image Vis. Comput. 30, (2012). doi:10.1016/j.imavis.2012.06.005

46. Sariyanidi, E., Gunes, H., Cavallaro, A.: Automatic analysis of facial affect: A survey of registration, representation, and recognition. IEEE Trans. Pattern Anal. Mach. Intell. 37, (2015). doi:10.1109/TPAMI.2014.2366127

47. Lopes, A.T., de Aguiar, E., De Souza, A.F., Oliveira-Santos, T.: Facial expression 
recognition with Convolutional Neural Networks: Coping with few data and the training sample order. Pattern Recognit. 61, (2017). doi:10.1016/j.patcog.2016.07.026

48. Mandal, M., Poddar, S., Das, A.: Comparison of human and machine based facial expression classification. In: International Conference on Computing, Communication and Automation, ICCCA 2015 (2015)

49. Samal, A., Iyengar, P.A.: Automatic recognition and analysis of human faces and facial expressions: a survey. Pattern Recognit. 25, (1992). doi:10.1016/00313203(92)90007-6

50. Taigman, Y., Yang, M., Ranzato, M., Wolf, L.: DeepFace: Closing the gap to humanlevel performance in face verification. In: Proceedings of the IEEE Computer Society Conference on Computer Vision and Pattern Recognition (2014)

51. Mukadam, N., Cooper, C., Kherani, N., Livingston, G.: A systematic review of interventions to detect dementia or cognitive impairment. Int. J. Geriatr. Psychiatry. 30, (2015). doi:10.1002/gps.4184

52. Petersen, R.C., Stevens, J.C., Ganguli, M., Tangalos, E.G., Cummings, J.L., DeKosky, S.T.: Practice parameter: Early detection of dementia: Mild cognitive impairment (an evidence-based review). Neurology. 56, (2001) 